received an anxiolytic (lorazepam) and 113 (54.1\%) received intravenous fluids. An antipsychotic (olanzapine) was administered to one patient $(0.5 \%)$. There were no reports of suicidal ideation, but physical restraints for agitation were employed in 13 patients (6.2\%). Police consultation occurred in 10 cases (4.8\%). No formal psychiatric consultations were requested by ED providers.

Discussion: Patients presenting to the emergency department from mass gathering events frequently endorse behavioral complaints requiring directed use of diagnostic and other emergency department resources for their ailments. The need for physical restraints and limited use of anxiolytics and antipsychotics in our sample suggest that psychiatric consultation is underutilized.

Prehosp Disaster Med 2019;34(Suppl. 1):s105-s106

doi:10.1017/S1049023X19002206

\section{The Birth and Growth of the National Ambulance Service in Ghana}

Mr. Mawuli Kingsley

National Ambulance Service, Nkawkaw, Ghana

Introduction: This study aimed to document the growth and challenges encountered in the decade since inception of the National Ambulance Service (NAS) in Ghana, West Africa. By doing so, potentially instructive examples for other low- and middle-income countries (LMICs) planning a formal prehospital care system or attempting to identify ways to improve existing emergency services could be identified.

Methods: Data routinely collected by the Ghana NAS from 2004-2014 were described, including: patient demographics, reason for the call, response location, target destination, and types of service. Additionally, the organizational structure and challenges encountered during the development and maturation of the NAS were reported.

Results: In 2004, the NAS piloted operations with 69 newly trained emergency medical technicians (EMTs), nine ambulances, and seven stations. The NAS expanded service delivery with 199 ambulances at 128 stations operated by 1,651 EMTs and 47 administrative and maintenance staff in 2014. In 2004, nine percent of the country was covered by NAS services; in 2014, $81 \%$ of Ghana was covered. Health care transfers and roadside responses comprised the majority of services ( $43 \%-80 \%$ and $10 \%-57 \%$ by year, respectively). Increased mean response time, stable case holding time, and shorter vehicle engaged time reflect greater response ranges due to increased service uptake and improved efficiency of ambulance usage. Specific internal and external challenges with regard to NAS operations also were described.

Discussion: The steady growth of the NAS is evidence of the need for Emergency Medical Services and the effects of sound planning and timely responses to changes in program indicators. The way forward includes further capacity building to increase the number of scene responses, strengthening ties with local health facilities to ensure timely emergency medical care and appropriateness of transfers, assuring a more stable funding stream, and improving public awareness of NAS services.

Prehosp Disaster Med 2019;34(Suppl. 1):s106

doi:10.1017/S1049023X19002218

\section{Blockchain Technology for Disaster and Refugee Relief Operations \\ Dr. Joseph McIsaac ${ }^{1,2}$, Mr. Joseph Brulle ${ }^{2}, M r$. John Burg' ${ }^{2}$, Mr. Gregory Tarnacki ${ }^{2}, M r$. Christian Sullivan ${ }^{2}$, Mr. Rick Wassel ${ }^{2}$ \\ 1. University Of Connecticut, Avon, United States \\ 2. Oxford Blockchain Strategy Programme, Saïd Business School, Oxford University, Oxford, United Kingdom}

Introduction: Blockchain is a distributed ledger technology for storing and transmitting information (value) that is secure, verifiable, and auditable. Two specific use-case opportunities exist, identity management and payment systems.

Aim: A secure and auditable solution for disaster refugee support.

Methods: Gap analysis, literature search, and synthesis using existing technologies.

Results: Strategy foundation: A blockchain identity management system that utilizes the Hyperledger Fabric framework; identification on a large scale, in a distributed model that provides immutable record capabilities to prevent fraud, with the ability to incorporate biometrics and DNA; deploy applications that will provide supply-chain capabilities; cryptocurrency for recipients and other relief functions for refugees/ disaster victims; components such as consensus, membership services, and Smart Contracts; cloud-based, with redundancies in multiple vendors and additional complex government cloud requirements/certifications, leveraging NIST $800-53$ by utilizing a hybrid public permissions architecture.

Discussion: There are an estimated 68 million refugees worldwide at any time. Valid identification is needed by most refugees to qualify for government or international donor relief. That identification is crucial in getting refugees and victims access to the aid supply chain. Blockchain stores data on a large number of computer nodes connected over the Internet. Each node contains an identical copy that is time-stamped and protected by a cryptographic technique called hashing, and control is decentralized. This blockchain strategy will revolutionize the way the government manages the $\$ 30$ billion in foreign aid to refugees. It will build upon the identities established to deploy applications that will provide supplychain capabilities, cryptocurrency for recipients, and other relief functions for refugees/disaster victims. Stakeholders beyond government will also benefit tremendously. The distributed nature of our application will provide visibility to NGOs, nonprofits, host nation stakeholders, and other relief organizations. A single system that provides information to everyone involved will almost instantaneously change the face of relief.

Prehosp Disaster Med 2019;34(Suppl. 1):s106

doi:10.1017/S1049023X1900222X 